\title{
A novel missense mutation in ACTG1 causes dominant deafness in a Norwegian DFNA20/26 family, but $A C T G 1$ mutations are not frequent among families with hereditary hearing impairment
}

\author{
Nanna D Rendtorff ${ }^{1}$, Mei Zhu ${ }^{2}$, Toril Fagerheim ${ }^{3}$, Torben L Antal ${ }^{4}$, MaryPat Jones ${ }^{5}$, \\ Tanya M Teslovich ${ }^{6}$, Elizabeth M Gillanders ${ }^{5}$, Michael Barmada ${ }^{7}$, Erik Teig ${ }^{8}$, \\ Jeffrey M Trent ${ }^{5,9}$, Karen H Friderici ${ }^{2}$, Dietrich A Stephan ${ }^{5,6,9}$ and Lisbeth Tranebjærg ${ }^{\star, 1,3,10}$ \\ ${ }^{1}$ Department of Medical Biochemistry and Genetics, Wilhelm Johannsen Centre for Functional Genomics, University of \\ Copenhagen, Copenhagen, Denmark; ${ }^{2}$ Department of Microbiology and Molecular Genetics, Michigan State University, \\ East Lansing, MI, USA; ${ }^{3}$ Department of Medical Genetics, University Hospital, Troms $\varnothing$, Norway; ${ }^{4}$ The Kinase Signalling \\ Laboratory, Biotech Research and Innovation Centre, Copenhagen, Denmark; ${ }^{5}$ Cancer Genetics Branch, National Human \\ Genome Research Institute, National Institutes of Health, Bethesda, MD, USA; ${ }^{6}$ Institute of Genetic Medicine, Johns Hopkins \\ University, Baltimore, MD, USA; ${ }^{7}$ Department of Human Genetics, University of Pittsburgh, Pittsburgh, PA, USA; \\ ${ }^{8}$ Department of Otorhinolaryngology, University Hospital of Oslo, Oslo, Norway; ${ }^{9}$ Neurogenomics Division, Translational \\ Genomics Research Institute, Phoenix, AZ, USA; ${ }^{10}$ Department of Audiology, H:S Bispebjerg Hospital, Copenhagen, Denmark
}

The $\gamma$-actin gene ( $A C T C 1)$ encodes a major cytoskeletal protein of the sensory hair cells of the cochlea. Recently, mutations in ACTG1 were found to cause autosomal dominant, progressive, sensorineural hearing impairment linked to the DFNA20/26 locus on chromosome 17q25.3 in four American families and in one Dutch family. We report here the linkage of autosomal dominant, progressive, sensorineural hearing impairment in a large Norwegian family to the DFNA20/26 locus. Sequencing of ACTG1 identified a novel missense mutation (c.1109T $>$ C; p.V370A) segregating with the hearing loss. Functional analysis in yeast showed that the p.V370A mutation restricts cell growth at elevated temperature or under hyperosmolar stress. Molecular modelling suggested that the p.V370A mutation modestly alters a site for protein-protein interaction in $\gamma$-actin and thereby modestly alters $\gamma$-actin-based cytoskeletal structures. Nineteen Norwegian and Danish families with autosomal, dominant hearing impairment were analyzed for mutations in ACTC 1 by sequencing, but no disease-associated mutations were identified. Finally, a longterm follow-up of the hearing loss progression associated with the p.V370A mutation in ACTG1 is provided. The present study expands our understanding of the genotype-phenotype relationship of this deafness gene and provides a sensitive and simple functional assay for missense mutations in this gene, which may assist future molecular diagnosis of autosomal-dominant hearing impairment. Finally, the present results do not indicate that mutations in ACTG1 are a frequent cause of autosomal-dominant postlingual sensorineural hearing impairment in Norway nor Denmark.

European Journal of Human Genetics (2006) 14, 1097-1105. doi:10.1038/sj.ejhg.5201670; published online 14 June 2006

Keywords: ACTC1; DFNA20/26; deafness; hereditary; late-onset; progressive

*Correspondence: $\operatorname{Dr}$ L Tranebjærg, Department of Audiology, $\mathrm{H}: \mathrm{S}$ Bispebjerg Hospital, Bispebjerg Bakke 23, DK-2400 Copenhagen NV, Denmark. Tel: + 4535 316341; Fax: + 4535 313951; E-mail: tranebjaerg@imbg.ku.dk

Received 9 November 2005; revised 24 April 2006; accepted 25 April 2006; published online 14 June 2006
Introduction

Non-syndromic hearing impairment (NSHI) is the most frequent sensory defect in humans and shows a very high degree of genetic heterogeneity. ${ }^{1}$ More than 100 genes are 
implicated in this disorder. ${ }^{2}$ At present, 54 chromosomal loci for autosomal-dominant NSHI, 60 loci for autosomal-recessive NSHI and seven loci for X-linked NSHI have been published or reserved. ${ }^{2}$ For these loci, 21 genes associated with autosomaldominant NSHI, 23 genes associated with autosomal-recessive NSHI and one gene associated with X-linked hearing impairment have been identified. ${ }^{2}$ These so-called deafness genes encode a large variety of proteins, which function in various cell types, structures and processes in the cochlea. ${ }^{3,4}$ The large diversity of deafness genes makes molecular diagnosis of hereditary hearing loss (HL) a difficult task.

Autosomal-dominant HL is estimated to account for $20 \%$ of hereditary deafness and, in contrast to autosomal-recessive $\mathrm{HL}$, shows a great variation in age of onset, rate of progression, severity of the hearing impairment and frequencies affected. ${ }^{5}$ It is therefore hoped that in future molecular diagnosis, the particular HL phenotype of a patient can help guide the selection of a limited number of genes for mutational analysis. ${ }^{5,6}$ This approach is already practised in the case of the WFS1-associated autosomal-dominant lowtone hearing impairment. ${ }^{7}$ The further success of this approach will be greatly facilitated by the identification and characterization of additional deafness genes/mutations and their associated audiological phenotypes.

Recently, mutations in ACTG1 were shown to cause autosomal-dominant hearing impairment linked to the DFNA20/26 locus (OMIM 604717) on chromosome 17q25.3. Five different HL-causing missense mutations in ACTG1 were described in four American families ${ }^{8}$ and in one Dutch family. ${ }^{9}$ ACTG1 encodes $\gamma$-actin, which is one of six highly conserved actin proteins in human. Actin monomers polymerize to form actin filaments, which are major structural components of the cytoskeleton and which regulate cell shape, cell motility, cell contraction and cell growth. Four actins are muscle specific, whereas $\beta$-actin and $\gamma$-actin are ubiquitously expressed. Notably, $\gamma$-actin is the predominant actin only in the auditory hair cells of the cochlea and in intestinal epithelial cells. ${ }^{10,11}$ The distinct expression pattern of $\gamma$-actin compared to other actins is thought to account for the non-syndromic HL phenotype caused by mutations in this gene. ${ }^{8}$

In order to elucidate the genetic basis of dominant $\mathrm{HL}$, we linked autosomal, progressive, sensorineural dominant NSHI in a large Norwegian family to chromosome 17q25.3 and identified a novel missense mutation (p.V370A) in ACTG1 segregating with the HL. We applied molecular modelling and a cellular assay to investigate whether the mutation may impair $\gamma$-actin function and evaluated whether $\gamma$-actin mutations are a frequent cause of HL.

\section{Materials and methods} Subjects and DNA isolation

A large Norwegian family (Figure 1a) and probands from five Norwegian and 14 Danish families with autosomal- dominant hearing impairment (2-5 generations affected, with at least four hearing-impaired family members details are available upon request) were investigated. None of these families had a specific genetic cause established or were pre-selected based on audiological patterns before inclusion in the study. In addition, eight probands from families with autosomal-recessive or sporadic occurrence of hearing impairment were studied. Genomic DNA was extracted from peripheral blood samples from the HL families as well as from 97 normal hearing individuals (from the same region in Norway as the large family studied) using standard methods. Blood samples were obtained after written consent.

The large Norwegian family was originally described by Teig. ${ }^{12}$ We included additional family members and reexamined previously characterized family members; so in total, 40 family members in four generations participated in this study. The follow-up time thereby became 41 years for some hearing-impaired family members. Two of the spouses in the family were hearing impaired: patient VI-2 has profound hearing impairment owing to Rhesus incompatibility, and patient V-1 belongs to a kindred of 11 sibs of whom five were profoundly hearing impaired from birth, although the parents had normal hearing thereby suggesting a recessive inheritance. The fact that his normally hearing parents were first cousins further supports this assumption. Thus, patients VI- 2 and V-1 were considered phenocopies and unaffected with respect to DFNA20/26. All affected individuals underwent pure-tone audiometry.

\section{Genome-wide linkage scan}

Genome-wide linkage analysis in the family described by Teig $^{12}$ was performed by amplifying 407 microsatellite markers built on a backbone of the ABI Prism Linkage Mapping Set, version 2 (Applied Biosystems). The average inter-marker distance was $8.66 \mathrm{cM}$. The PCR products were amplified in $15 \mu \mathrm{l}$ reactions containing $50 \mathrm{ng}$ of genomic DNA, $0.33 \mu \mathrm{M}$ of each primer, $0.25 \mu \mathrm{M}$ of each dNTP, $2.5 \mathrm{mM}$ $\mathrm{MgCl}_{2}, 10 \mathrm{mM}$ Tris- $\mathrm{HCl}, 50 \mathrm{mM} \mathrm{KCl}$ and $0.5 \mathrm{U}$ Taq polymerase. PCR amplifications were performed in a 9600 or 9700 thermocycler (Applied Biosystems) with the following cycling parameters: $12 \mathrm{~min}$ at $95^{\circ} \mathrm{C} ; 10$ cycles of $94^{\circ} \mathrm{C}$ for $45 \mathrm{~s}, 55^{\circ} \mathrm{C}$ for $1 \mathrm{~min}$ and $72^{\circ} \mathrm{C}$ for $1 \mathrm{~min} ; 20$ cycles of $89^{\circ} \mathrm{C}$ for $1 \mathrm{~min}, 55^{\circ} \mathrm{C}$ for $1 \mathrm{~min}$ and $72^{\circ} \mathrm{C}$ for $1 \mathrm{~min} ; 10 \mathrm{~min}$ final extension at $72^{\circ} \mathrm{C}$. PCR products were pooled based on fluorescent labels and expected allele size range, and electrophoresed through 96-lane 5\% denaturing polyacrylamide gels on Applied Biosystems 377 sequencers. The GENESCAN and GENOTYPER software packages (Applied Biosystems) were used for allele sizing and binning. After linkage was demonstrated to $17 q 25$, additional markers (Table 1, online Supplementary material) in this region were genotyped in order to narrow the genetic 


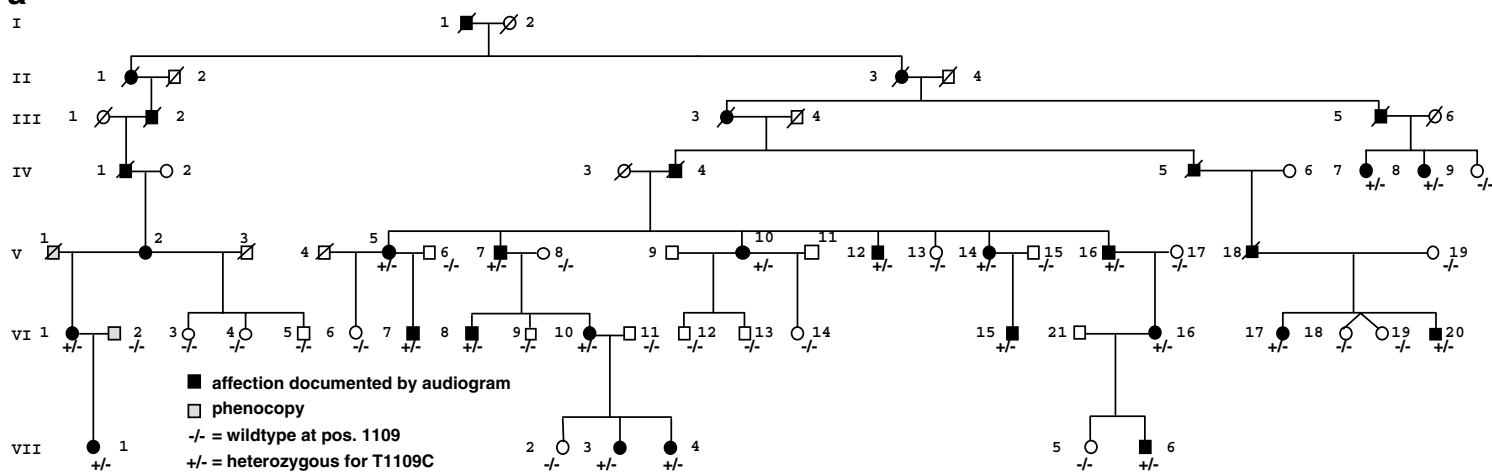

b

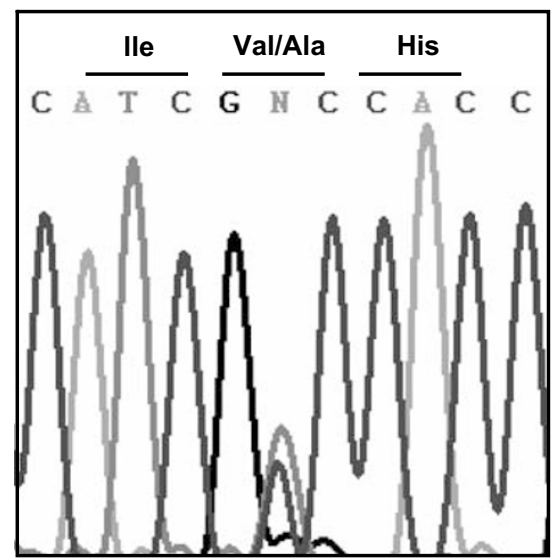

Patient

C

C Frequency $(\mathrm{Hz})$

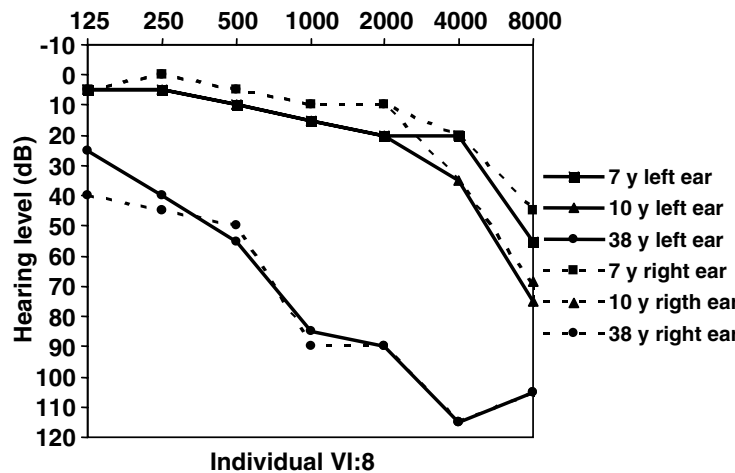

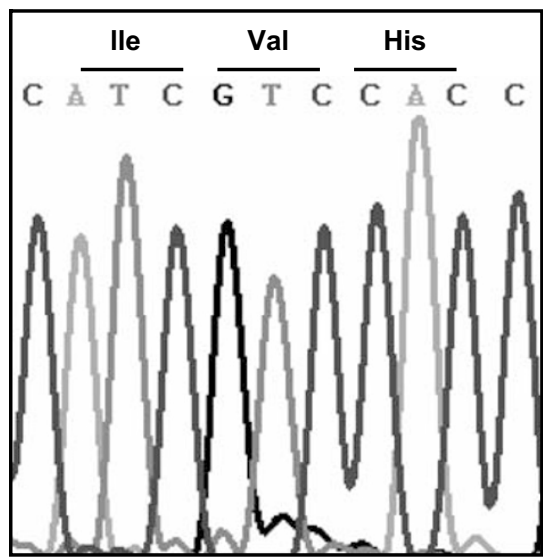

Control

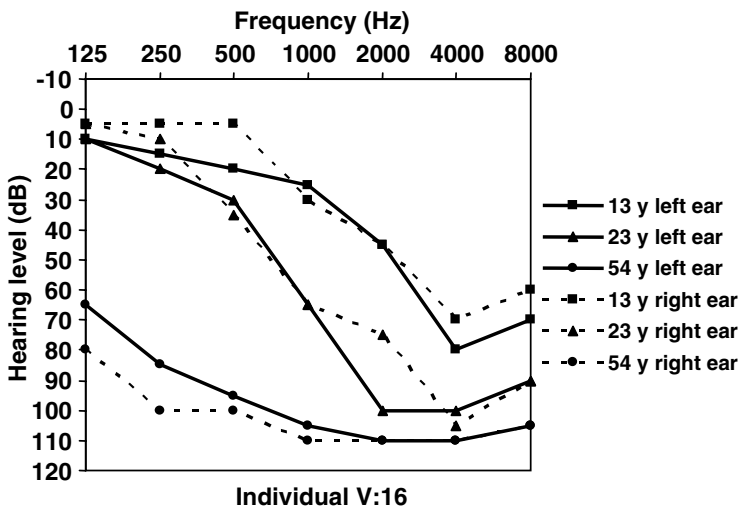

Figure 1 (a) Pedigree of the Norwegian DFNA20/26 family with autosomal-dominant progressive hearing impairment and segregation of the ACTC 1 c. 1109 T > C missense mutation. Family members tested positive for the missense mutation are indicated by $+/-$, and family members tested negative for the mutation are indicated by $-/-$. (b) $\gamma$-Actin mutation analysis. Sequencing result showing the c.1109T $>C$ mutation in $A C T G 1$ exon 6 . The mutation is heterozygous in affected individuals. (c) Audiograms of left and right ear of representative, affected family members (patients VI-8 and $\mathrm{V}$-16). The age at the time of analysis is indicated. The observation period was 31 and 41 years in patients VI-8 and V-16, respectively.

region. Both two-point linkage and multipoint linkage analyses were performed using Vitesse version $1.1 .^{13}$

\section{ACTG1 sequence analysis}

PCR for ACTG1 sequencing in the large Norwegian family was performed on DNA from two affected and two non- affected family members using primers described by Zhu et $a .^{8}$ Following identification of the c.1109T $>\mathrm{C}$ mutation, all family members were sequenced in position c.1109. PCR for ACTG1 sequencing in affected family members from 20 small Norwegian and Danish families segregating autosomal-dominant hearing impairment was 
performed by amplifying the coding region (exons 2-6) of $A C T G 1$ as one PCR product $(1840 \mathrm{bp})$ using the Expand ${ }^{\text {TM }}$ Long Template PCR system enzyme mix (Roche Applied Science) and primers 5'-CTTCCCCGCGGGAGGGCAT TAGGT-3' and 5'-GCATGAGGTGTGTGCATTTGCCAGG$3^{\prime}$. Thermocycler conditions were an initial denaturation at $95^{\circ} \mathrm{C} ; 40$ cycles of $94^{\circ} \mathrm{C}$ for $20 \mathrm{~s}, 63^{\circ} \mathrm{C}$ for $30 \mathrm{~s}$ and $68^{\circ} \mathrm{C}$ for $3 \mathrm{~min}$; with a final extension for $7 \mathrm{~min}$ at $68^{\circ} \mathrm{C}$. This PCR product was then used as template for exon-by-exon sequencing. Primer sequences are available from the authors upon request. PCR-amplified samples were sequenced by using ${ }^{33} \mathrm{P}$ and the Thermo Sequenase Cycle Sequencing Kit (Amersham Life Science) or by using Big Dye Terminator ${ }^{\mathrm{TM}}$ Cycle Sequencing Ready Reaction Kit according to the manufacturer's instructions.

\section{Analysis of DNA from normal controls for the c.1109C $>$ T mutation}

A 258-bp PCR product containing position c.1109 of ACTG1 was PCR-amplified in $25 \mu \mathrm{l}$ using the primers $5^{\prime}$ GTGCCTGGCTGTCTTTGCAGA-3' and 5'-CGTGAGGC TAGCATGAGGTGTG-3'. Thermocycler conditions were an initial denaturation at $94^{\circ} \mathrm{C}$ for $5 \mathrm{~min}$; 40 cycles of $94^{\circ} \mathrm{C}$ for $20 \mathrm{~s}, 60^{\circ} \mathrm{C}$ for $20 \mathrm{~s}$ and $72^{\circ} \mathrm{C}$ for $30 \mathrm{~s}$; with a final extension for $5 \mathrm{~min}$ at $72^{\circ} \mathrm{C}$. Fifteen microlitres of the PCR sample was digested overnight with $2 \mathrm{U} H$ py8I (Fermentas) in a $30-\mu$ l reaction and analysed on a $2 \%$ agarose gel.

\section{Molecular modelling}

A model of human $\gamma$-actin residues $2-375$ was generated using the homology modules in INSIGHT II (2001; Biosym/ MSI). The template used for the modelling was the crystal structure coordinates of bovine $\beta$-actin crystallized in a complex with human profilin (PDB accession number $\left.2 \mathrm{BTF},{ }^{14}\right)$. The program GRID, ${ }^{15}$ version 21 , Molecular Discovery Ltd, was used to compute hydrophobic surface contours in the model of $\gamma$-actin in order to predict residues in $\gamma$-actin that engage in hydrophobic interactions with V370. Figures were prepared using the program PyMOL, version 0.97 (http://www.pymol.org).

\section{Yeast growth assay}

Plasmid pRSWT and yeast strain PR1 were gifts from Dr Peter Rubenstein (University of Iowa, USA). The p.V370A missense change was introduced into the actin gene in plasmid pRSWT by site-directed mutagenesis. After transformation of the wild-type or mutant plasmid into yeast (PR1) and selection with 5 -fluoroorotic acid, the yeast cultures were grown in TRP selection medium at $30^{\circ} \mathrm{C}$ overnight, and then grown in YPD medium for $6 \mathrm{~h}$ with starting $\mathrm{OD}_{600}=0.1$. The cells were then plated on agar plates with YPD or YPD $+0.5 \mathrm{M} \mathrm{NaCl}$ at $5,5 \times 10,5 \times 10^{2}$ or $5 \times 10^{3}$ cells/spot. The cells were incubated at 30 and $37^{\circ} \mathrm{C}$ for 3 days and then analysed.

\section{Results \\ Linkage to the DFNA20/26 locus}

Before the genome-wide screen in the family described by Teig, ${ }^{12}$ we investigated possible linkage to various known NSHI loci (DFNA2-DFNA7, DFNA10, DFNB2, DFNB6, DFNB8 and DFNB10), but linkage to any of these loci could be excluded (data not shown). We also sequenced GJB2 in seven affected (VI:20, VI:17, V:16, VI:10, VI:2, VI:1 and IV:7) and two (VI:18 and V:19) normal hearing family members because of its prevailing aetiological role in hereditary, including dominantly inherited deafness (http://davinci.crg.es/deafness/). Individuals VI-17 (affected) and V-19 (unaffected) were found to be heterozygous for the p.M34T missense change. No other GJB2 mutations or polymorphisms were identified. The lack of co-segregation between the p.M34T allele and the hearing impairment indicates that this mutation does not play a major role in HL in the family.

A genome-wide scan identified a putative locus on chromosomes 16 and 17 . The region on chromosome 16 between markers D16S3075 and D16S415 (maximum LOD score of 1.7) was analysed with additional markers (D16S519, D16S3041, D16S420, D16S690, D16S3396 and D16S771) but negative LOD scores were obtained for these markers. In contrast, genotyping of additional markers (D17S1807, D17S802 and D17S668) at chromosome 17q25 strengthened linkage to this region with a maximum twopoint LOD score of 5.47 (Theta $=0)$ at marker D17S668. Multipoint linkage analysis gave a strong peak (multipoint LOD score of 9.16) between markers D17S784 and D17S928. By examination of haplotypes using genotypes of five markers, the minimum candidate region, limited by recombination events in hearing-impaired individuals, is flanked by markers D17S784 and D17S928 representing a distance of $6.1 \mathrm{cM}$ on the deCode genetic map. Linkage to this chromosomal region has previously been reported for autosomal-dominant $\mathrm{HL}$ and two overlapping hearing impairment locus numbers, DFNA20 and DFNA26, have been assigned to the region. ${ }^{16,17}$ Recently, ACTG1 was found to be the causative gene for hearing impairment in this region in five American and Dutch families (Table 2, online Supplementary material). ${ }^{8,9}$

\section{Mutation analysis of $A C T G 1$}

In order to determine whether $A C T G 1$ is mutated in the family described by Teig, ${ }^{12}$ sequencing of the ACTG1 coding region (GenBank no. X04098.1 (mRNA sequence) and GenBank no. M19283 (genomic sequence)) in two affected and two unaffected members of this family was performed. The analysis identified a potentially diseasecausing c.1109T $>$ C nucleotide transition in exon 6 of the affected family members, which results in substitution of alanine for valine in position 370 of $\gamma$-actin (Figure 1b). DNA from 19 affected and 21 non-affected family members was thereafter analysed and the mutation showed perfect 
co-segregation with the HL in all 19 affected individuals. Furthermore, 97 normally hearing individuals originating from the same geographic location in Norway (Trøndelag, Mid-Norway) as the present family were analyzed for the mutation. This analysis was performed by Hpy8I digestion of PCR-amplified genomic DNA, as the T-to- $C$ transition of the mutation destroys a cleavage site for this endonuclease. The mutation was not found in any of the normally hearing individuals. V370 is conserved in all human actin isoforms as well as in actins from mouse, fruitfly, worm, yeast and slime mold. The evolutionary conservation of V370, the co-segregation of the p.V370A mutation with HL and the absence of the mutation in normal hearing control individuals strongly indicated that this mutation underlies the HL observed in the Norwegian family.

The p.V370A mutation impairs growth of yeast cells In order to test directly whether the p.V370A mutation impairs actin function, we performed growth assays with yeast (Saccharomyces cerevisiae) expressing wild-type yeast actin or yeast actin with the presumed deafness mutation. Yeast expresses only one actin, which is $89 \%$ identical to human $\gamma$-actin and in which the valine is conserved at amino-acid position 370. The p.V370A mutation was found to affect growth of yeast only slightly under normal growth conditions, that is, growth on YPD agar plates at $30^{\circ} \mathrm{C}$ (Figure 2a). By contrast, the p.V370A mutation severely impaired growth during hyperosmolar stress (Figure 2b) as well as growth at elevated temperature
(Figure 2c). When both stresses were applied together, the p.V370A mutation completely suppressed growth (Figure 2).

\section{Structural consequences of the p.V370A mutation}

We next investigated the structural consequences of the p.V370A mutation. Owing to a high degree of sequence conservation (99\% identity), the crystal structure coordinates of bovine $\beta$-actin, complexed with the actin-regulatory protein profilin, could be used to generate a reliable model of human $\gamma$-actin using the Inside homology modelling program (Figure 3). Analysis of the model using the program GRID, ${ }^{15}$ showed that the side chain of V370 engages in hydrophobic interactions with the side chains of Arg116, Val134, His371 and Cys374 (Figure 3b) and these interactions appear to be a major determinant in stabilizing the position of the C-terminus of $\gamma$-actin (residues 368-375). Superimposition of several actin structures from various species ${ }^{18-21}$ indicated that V370 has a conserved role in stabilizing the position of the $\mathrm{C}$ terminus of actins via these hydrophobic interactions (data not shown). Importantly, analysis of a model of the p.V370A mutant revealed that the side chain of A370 is too short to establish hydrophobic interactions with Arg116, Val134, His371 and Cys374 (Figure 3c). Otherwise, the p.V370A mutation is not predicted to alter the structure of $\gamma$-actin. Thus, molecular modelling analysis strongly suggested that the p.V370A mutation affects $\gamma$-actin function by destabilizing the position of the C-terminal tail.
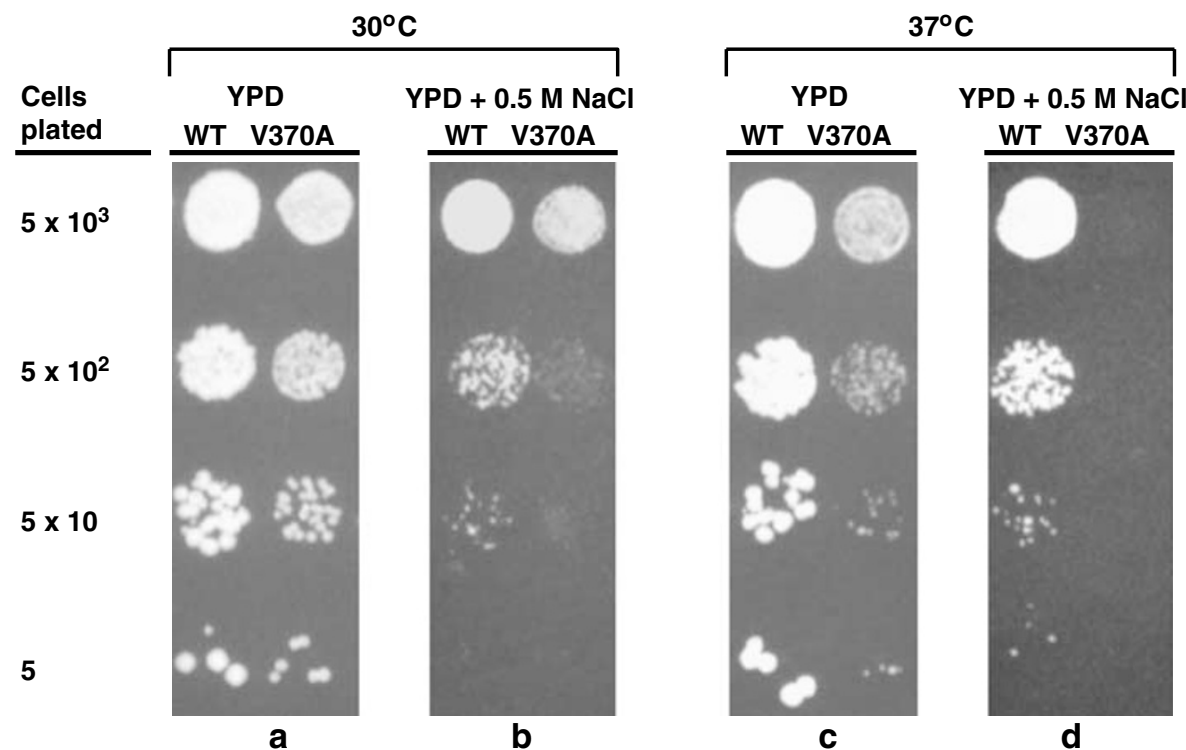

Figure 2 Yeast growth assay. Yeast cells expressing either wild-type or p.V370A mutant $\gamma$-actin were plated on YPD or YPD $+0.5 \mathrm{M} \mathrm{NaCl}$ agar plates at various densities and incubated at 30 or $37^{\circ} \mathrm{C}$. The photograph shows the cultures 3 days after plating. The experiments were repeated three times with similar results. 

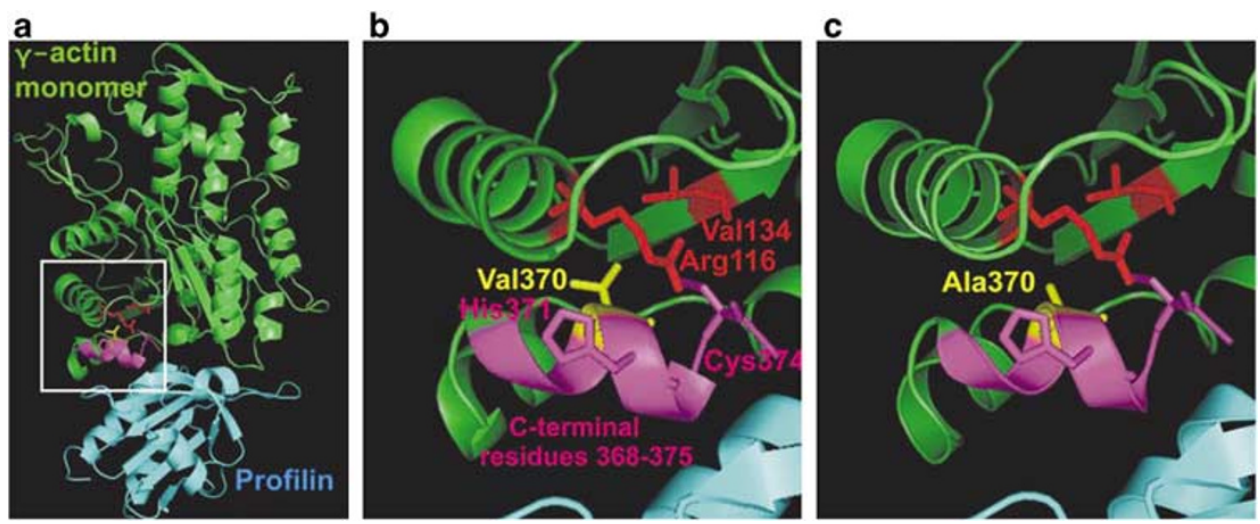

Figure 3 Modelling of $\gamma$-actin. (a) Structural model of wild-type $\gamma$-actin (green) in complex with profilin (blue) shown as ribbon representations with side chains of residues discussed in the text. V370 is shown in yellow, and the C-terminus of $\gamma$-actin and residues herein that interact with V370 in magenta. Residues elsewhere in $\gamma$-actin that interact with V370 are shown in red. (b) Enlarged view of the boxed region in (a). (c) As in (b), except that $\gamma$-actin is the p.V370A mutant.

\section{Phenotype of HL associated with the p.V370A mutation}

The present family shows non-syndromic, autosomaldominant hearing impairment with complete penetrance and variable onset. The hearing-impaired individuals present bilateral, sloping, progressive sensorineural hearing impairment. Based on 19 persons belonging to the three youngest generations, the average age for diagnosis of hearing impairment was 35.3 years. However, the average age of onset of hearing impairment is surely much lower, as, particularly older, members of this family living in a remote valley in mid-Norway tended to seek medical help for diagnosis of hearing impairment fairly late. Considering mainly the younger members of the family, the age of diagnosing hearing impairment ranged from age 6 (in VII:6) to age 24 years (in VII:1). We estimate that the average age of onset of hearing impairment is in the first or second decade. In agreement with this estimate, most of the affected members of the family needed hearing aid from about age 20 years. As described by Teig, ${ }^{12}$ the HL first affects the highest frequencies, frequently around 7 years of age, and progresses to $60-80 \mathrm{~dB} \mathrm{HL}$ for frequencies $>1000 \mathrm{~Hz}$. It then progresses to a $40-60 \mathrm{~dB} \mathrm{HL}$ for frequencies $<1000 \mathrm{~Hz}$. Further progression leads to profound hearing impairment for frequencies $>1000 \mathrm{~Hz}$ in the majority of the affected individuals, gradually presenting as a flat or corner audiogram. Our subsequent audiological examination of the family is in very good agreement with the findings by Teig. ${ }^{12}$ Figure $1 \mathrm{c}$ shows two representative audiograms from the family, which illustrate the progression of the HL over 31 and 41 years, respectively, thereby providing a long-term follow-up time of examination. In Supplementary Figure 1 is shown an audiogram of the earliest documented age of onset of hearing impairment in the family, which was at 6 years of age. As reported by Teig, ${ }^{12}$ the development of HL generally followed a similar pattern in affected individuals. In the present study, however, we noted examples of significant variation in the rate of progression of HL, as illustrated in Supplementary Figure 1, showing audiograms from two female subjects recorded at similar ages. We were not able to determine whether gender affected the HL phenotype.

Three affected family members (patients V-12, V-16 and VI-15) received a cochlear implant at the ages of 63,58 and 43 years, respectively, but had limited benefit from the device. However, they also did not attend audiological speech rehabilitation and kept being primarily sign language users despite being able to sense sound after the implantation. Our observations were too anecdoctal to determine if their limited benefit of cochlear implant was due to lack of compliance with speech rehabilitation or indicated a degenerative component affecting central auditory pathways.

As described above, individual VI:17 had a p.M34T GJB2 mutation in addition to the ACTG1 mutation, raising the question whether this individual might display a more severe HL due to the double mutation. However, individual VI:17 had at age 36 years a $\mathrm{PTA}_{0.25-8 \mathrm{kHz}}$ (puretone average audiogram over the frequencies $0.25-8 \mathrm{kHz}$ ) of $41 / 36 \mathrm{~dB} \mathrm{HL}$ for left/right ear, whereas her hearingimpaired brother (VI:20), also with the ACTG1 mutation but with normal GJB2 sequence, had a $\mathrm{PTA}_{0.25-8 \mathrm{kHz}}$ of $58 / 62 \mathrm{~dB} \mathrm{HL}$ left/right ear at age 31 years. To further investigate the p.M34T GJB2 mutation, we performed protein expression analysis of connexin 26 (the protein encoded by GJB2) and 43 in cultured skin keratinocytes from a skin biopsy of individual VI:17 as well as of VI:18 and V:19 (with normal GJB2 sequence), as described. ${ }^{22}$ This showed immunohistochemical signals of similar intensity for both connexin 26 and 43 in keratinocytes from all three individuals (data not shown). Taken together, these results indicate that the p.M34T GJB2 mutation is not likely to affect the deafness phenotype of the p.V370A ACTG1 mutation. 
Vestibular dysfunction, manifested as some equilibristic instability, was claimed by some of the elderly, profoundly hearing-impaired individuals, but was not formally tested in the family.

\section{Mutation analysis of $A C T G 1$ in families with unknown cause of autosomal-dominant hearing impairment}

Finally, we wished to elucidate whether mutations in ACTG1 may be a frequent cause of autosomal-dominant hearing impairment. The entire coding region and intronic splice junctions of ACTG1 were therefore sequenced in affected members from 19 small Norwegian and Danish families with dominantly inherited hearing impairment of unknown cause. In addition, eight probands from families with autosomal-recessive or sporadic occurrence of hearing impairment were analysed. In this analysis, we included families with autosomal-recessive $\mathrm{HL}$, because certain deafness genes (TMC1, TECTA, MYO7A) have been found to underlie autosomal-dominant as well as recessive $\mathrm{HL}$, and we therefore wished to investigate whether that might also be the case with ACTG1.

Six nucleotide changes were found in ACTG1 in the 27 families, but none of the changes are likely to cause HL (Table 3, online Supplementary material). In exon 5, the synonymous mutations c.918C $>$ T (ENTREZ SNP database 1139405) and c.930C $>$ T (ENTREZ SNP database 1135989) were heterozygously as well as homozygously present. A novel IVS1-3C $>\mathrm{T}$ variation was detected in one affected individual, but $\mathrm{T}$ is almost as frequent as $\mathrm{C}$ in position 3 before an exon (reviewed by Cartegni et $a l^{23}$ ), suggesting that the present variation does not affect splicing of exon 2 . The IVS3 $+13 \mathrm{C}>\mathrm{A}$ and IVS5 + 54delG variations were identified in several affected individuals, but have previously been reported not to segregate with HL associated with the DFNA20/26 locus. ${ }^{9}$ Finally, a synonymous change in the stop codon (ENTREZ SNP database 11549223) was detected.

\section{Discussion}

The present study extends the recent discovery of ACTG1 as a new autosomal-dominant deafness gene ${ }^{8,9}$ by identification and characterization of a novel ACTG1 missense mutation and the resulting HL phenotype. We also used a functional yeast assay that may help evaluate whether a missense mutation is pathogenic. Furthermore, we report a series of ACTG1 mutation-negative families, suggesting that ACTG1 mutations do not play a major causative role in the $20 \%$ of all hereditary HL that shows autosomaldominant inheritance.

Impairment of actin function owing to the p.V370A mutation was demonstrated in a yeast growth assay. These experiments, however, also indicated that the mutation does not drastically disrupt actin function, as growth was only significantly impaired when the yeast were cultured at elevated temperature or under osmotic stress. Molecular modelling supported the conclusion that the mutation does only modestly alter the function of $\gamma$-actin, as it was not predicted to globally alter the structure of $\gamma$-actin, but only affects the position of the C-terminal tail (about 6-8 residues). The effect of mutation of V370 in actins has not been reported previously, but other mutations in the $\mathrm{C}$ terminal tail have been shown to alter actin function to various degrees, ranging from minor effects to lethality in yeast growth assays (reviewed by Sheterline et $a l^{24}$ ). Many studies have suggested that the C-terminal tail of actins forms part of a binding site for important actin-binding proteins, including profilin, cofilin and gelsolin. These proteins act to either stimulate or counteract the formation of actin filaments and thereby regulate the cytoskeleton. ${ }^{24,25}$ In conclusion, the molecular modelling analysis therefore suggests that the p.V370A mutation may cause a modest alteration of actin-based structures, or of their dynamics of formation, in the cell.

In the cochlea, the most prominent actin-based structures are found in the hair cells. ${ }^{26}$ Thus, parallel actin filaments are key structural elements in determination of the shape and function of the stereocilia of the hair cells, which are the mechanosensors for sound waves. Moreover, in the hair cells, the stereocilia are anchored into the cuticular plate, which consists of a gel-like network of actin filaments. It is therefore likely that mutations in $\gamma$-actin cause HL primarily by impairing the function and/or viability of hair cells.

Interestingly, similar to the present p.V370A mutation, the five recently reported deafness-causing mutations in $\gamma$-actin are all missense mutations that are predicted to only modestly affect the structure and function of $\gamma$-actin. The predicted modest functional impairment at the molecular level may account for the late-onset $\mathrm{HL}$ phenotype associated with these missense mutations in ACTG1. According to this hypothesis, hair cells with modestly impaired actin structures (or dynamics whereby these are formed) are fully functional, as evidenced by normal juvenile hearing of individuals with these mutations. However, such hair cells may exhibit increased sensitivity to age-dependent degeneration. As hair cells do not regenerate, this degeneration ultimately results in lateonset, progressive HL. $\gamma$-Actin is also the predominant actin in intestinal epithelial cells. In contrast to hair cells, these cells are constantly regenerating, which may explain why missense mutations that only mildly impair $\gamma$-actin function result in a non-syndromic HL phenotype. By contrast, deletion or mutations that profoundly alter the structure of $\gamma$-actin are likely to be embryonic lethal owing to lethal effects on intestinal epithelial cells and/or other cell types, which may explain why such mutations have not been reported. It should be noted, however, that any, presumably mild, gastrointestinal phenotype associated with the $\gamma$-actin deafness mutations has not been ruled out at this point. 
Increased vulnerability to hair cell degeneration is the most likely cause of HL associated with missense mutations in ACTG1, and should indicate optimistic prognosis after cochlear implantation. The potential of this treatment may not be truly reflected in the relative poor outcome in the mutation carriers in the present study. The observation by Teig $^{12}$ of a pathological tone decay found in some frequences where the HL was more than $50 \mathrm{~dB}$, suggests, however, that missense mutations in ACTG1 might cause degenerative lesions in the central auditory pathways in addition to hair cell degeneration. Therefore, the benefit of a cochlear implant in DFNA20/26 patients deserves a more systematical evaluation including testing otoacoustic emissions.

All DFNA20/26 families, including the Norwegian family, display progressive, bilateral, sensorineural HL that begins at the high frequencies. ${ }^{8,27-29}$ As age increases, the degree of HL increases, with threshold shifts seen at all frequencies, although a sloping configuration is usually maintained. The final outcome is most often a 'corner audiogram' and profound HL. Differences between families and intersubject are seen in average age at onset of HL, which varies from the first decade to the third decade, and rate of progression, suggesting that the different types of ACTG1 mutations and/or differences in genetic and environmental background modify the phenotypes. In the Norwegian family, most of the hearing-impaired family members needed a hearing aid by age 20 years. The typical age of onset of HL is thus similar to three of the previously published DFNA20/ 26 families, where the hearing impairment was noticed in the second decade. In contrast, one family described by Kemperman et $a l^{29}$ displayed a more severe phenotype as the HL by age 15-20 years had become profound at $4-8 \mathrm{kHz}$, and by age 30-50 years, profound also at frequencies between 2 and $4 \mathrm{kHz}$. The maximum rate of progression occurred between 10 and 35 years of age with a progression rate of $3-8 \mathrm{~dB} /$ year, and by age $30-50$ years the final degree of residual hearing was reached. ${ }^{29}$

As ACTG1 missense mutations appear to sensitize hair cells to age-dependent degeneration, it can be speculated that such mutations may be involved in the widely occurring HL of the elderly, called presbyacusis. However, despite similarities between presbyacusis and $\gamma$-actinrelated hearing abnormality, the age of onset of $\gamma$-actinrelated hearing abnormality was 30 years earlier than presbyacusis, the rate of progression was faster and the degree to which mid-frequencies became affected with increasing age was much more pronounced. ${ }^{29}$ Moreover, the speech recognition was found to be better in DFNA20/ 26 individuals than in individuals with presbyacusis. ${ }^{29}$ These discrepancies may indicate that ACTG1 missense mutations may not be involved in presbyacusis to any significant extent.

The recent and present demonstrations of HL-causing mutations in ACTG1 in a total of six families originating from Europe and USA justified examination whether mutations in ACTG1 are a frequent cause of autosomal hearing impairment. However, we found no ACTG1 mutations in 19 Norwegian and Danish families segregating autosomal-dominant hearing impairment. Thus, although the number of families studied was relatively small, the present findings do not indicate that ACTG1 mutations are a predominant cause of hereditary hearing impairment with autosomal-dominant inheritance. Thus, so far, no single gene is reported to be responsible for any substantial fraction of autosomal-dominant hearing impairment.

\section{Acknowledgements}

We thank the patients who participated in this study. In addition, we thank Geir Fostad and Hans Omland, Department of Audiology, Levanger Hospital, Norway for audiological testing and Vigdis Brox, Department of Medical Genetics, University Hospital of Tromsø, Norway for help with genotyping. We acknowledge Nick Lench, Wales Gene Park, Medicentre, Cardiff, for GJB2 mutation testing. We would like to thank Professor Malcolm Hodgins, Department of Dermatology, University of Glasgow for the immunohistochemical connexin 26 expression studies in cultivated keratinocytes from skin biopsies. We acknowledge Danish Centre for Scientific Computing for access to SGI computers.This work was supported by the Oticon Foundation, Denmark and the National Human Genome Research Institute Intramural Program, USA.

\section{References}

1 Morton N: Genetic epidemiology of hearing impairment. Ann NY Acad Sci 1991; 630: 16-31.

2 Van Camp G, Smith R: Hereditary hearing loss homepage http:// dnalab-www.uia.ac.be/dnalab/hhh/ (August, 2005).

3 Steel KP, Kros CJ: A genetic approach to understanding auditory function. Nat Genet 2001; 27: 143-149.

4 Petit C, Levilliers J, Hardelin J-P: Molecular genetics of hearing loss. Ann Rev Genet 2001; 35: 589-646.

5 Bitner-Glindzicz M: Hereditary deafness and phenotyping in humans. Br Med Bull 2002; 63: 73-94.

6 Bischoff AM, Luijendijk MW, Huygen PL et al: A novel mutation identified in the DFNA5 gene in a Dutch family: a clinical and genetic evaluation. Audiol Neurootol 2004; 9: 34-46.

7 Smith RJ: Clinical application of genetic testing for deafness. Am J Med Genet A 2004; 130: 8-12.

8 Zhu M, Yang T, Wei S et al: Mutations in the gamma-actin gene (ACTG1) are associated with dominant progressive deafness (DFNA20/26). Am J Hum Genet 2003; 73: 1082-1091.

9 Van Wijk E, Krieger E, Kemperman $\mathrm{MH}$ et al: A mutation in the gamma actin 1 (ACTG1) gene causes autosomal dominant hearing loss (DFNA20/26). J Med Genet 2003; 40: 879-884.

10 Hofer D, Ness W, Drenckhahn D: Sorting of actin isoforms in chicken auditory hair cells. J Cell Sci 1997; 110: 765-770.

11 Vandekerckhove J, Leavitt J, Kakunaga T, Weber K: Coexpression of a mutant beta-actin and the two normal beta- and gammacytoplasmic actins in a stably transformed human cell line. Cell 1980; 22: 893-899.

12 Teig E: Hereditary progressive perceptive deafness in a family of 72 patients. Acta Oto-laryngol 1968; 65: 365-372.

13 O'Connell JR, Weeks DE: The VITESSE algorithm for exact multilocus linkage analysis via genotype set-recoding and fuzzy inheritance. Nat Genet 1995; 11: 402-408.

14 Schutt CE, Myslik JC, Rozycki MD, Goonesekere NCW, Lindberg U: The structure of crystalline profilin-beta-actin. Nature 1993; 365: $810-816$. 
15 Goodford PJ: A computational procedure for determining energetically favorable binding sites on biologically important macromolecules. J Med Chem 1993; 28: 849-857.

16 Morell RJ, Friderici KH, Wei S, Elfenbein JL, Friedman TB, Fisher RA: A new locus for late-onset, progressive, hereditary hearing loss DFNA20 maps to 17q25. Genomics 2000; 63: 1-6.

17 Yang T, Smith R: A novel locus DFNA26 maps to chromosome $17 \mathrm{q} 25$ in two unrelated families with progressive autosomal dominant hearing loss. Am J Hum Genet 2000; 67 (Suppl 2): 300.

18 McLaughlin PJ, Gooch JT, Mannherz H-G, Weeds AG: Structure of gelsolin segment 1-actin complex and the mechanism of filament severing. Nature 1993; 364: 685-692.

19 Otterbein LR, Cosio C, Graceffa P, Dominguez R: Crystal structures of the vitamin D-binding protein and its complex with actin: structural basis of the actin-scavenger system. Proc Natl Acad Sci USA 2002; 99: 8003-8008.

20 Klenchin VA, Allingham JS, King R, Tanaka K, Marriott G, Rayment I: Trisoxazole macrolide toxins mimic the binding of actin-capping proteins to actin. Nat Struct Biol 2003; 10: 1058-1063.

21 Hertzog M, van Heijenoort C, Didry D et al: The beta-thymosin/ WH2 domain; structural basis for the switch from inhibition to promotion of actin assembly. Cell 2004; 117: 611-623.
22 Rouan F, White TW, Brown N et al: Trans-dominant inhibition of connexin- 43 by mutant connexin-26: implications for dominant connexin disorders affecting epidermal differentiation. J Cell Sci 2001; 114: 2105-2113.

23 Cartegni L, Chew SL, Krainer AR: Listening to silence and understanding nonsense: exonic mutations that affect splicing. Nat Rev Genet 2002; 3: 285-298.

24 Sheterline P, Clayton J, Sparrow J: Actin. Protein Profile 1995; 2 : $1-103$.

25 Dominguez R: Actin-binding proteins - a unifying hypothesis. Trends Biochem Sci 2004; 29: 572-578.

26 Slepecky NB in Dallos P, Popper AN, Fay RR (eds): Structure of the Mammalian Cochlea. New York: The cochlea, 1996, pp 44-129.

27 Elfenbein JL, Fisher RA, Wei S et al: Audiological aspects of the search for DFNA20: a gene causing late-onset, progressive, sensorineural hearing loss. Ear Hear 2001; 22: 279-288.

28 DeWan AT, Parrado AR, Leal SM: A second kindred linked to DFNA20 (17q25.3) reduces the genetic interval. Clin Genet 2003; 63: $39-45$.

29 Kemperman MH, De Leenheer EMR, Huygen PLM et al: A Dutch family with hearing loss linked to the DFNA20/26 locus. Longitudinal analysis of hearing impairment. Arch Otolaryngol Head Neck Surg 2004; 130: 281-288.

Supplementary Information accompanies the paper on European Journal of Human Genetics website (http://www.nature.com/ejhg) 